\title{
Estudo da Cinética de Cura de um Pré-impregnado de Resina Epoxídica/Fibra de Carbono pelo Método Isoconversional
}

\author{
Vanesa C. G. M. Ferrari \\ Faculdade de Engenharias, Arquitetura e Urbanismo, Universidade do Vale do Paraíba - UNIVAP \\ Margarete F. P. Azevedo, Luis H. David, Vera L. Lourenço \\ Divisão de Química, Instituto de Aeronáutica e Espaço - IAE, Centro Técnico Aeroespacial - CTA
}

\begin{abstract}
Resumo: Este trabalho tem como objetivo verificar a cinética de cura de um pré-impregnado ("prepreg") de resina epoxídica/fibra de carbono, utilizando a técnica de calorimetria diferencial de varredura (DSC) pelo método isoconversional. O método isoconversional analisa a cinética de cura por meio da dependência da energia de ativação durante a conversão (análise isotérmica) ou temperatura (análise não-isotérmica) e utiliza esta dependência para calcular os parâmetros cinéticos. A avaliação da cinética da reação por DSC baseia-se na quantidade de calor gerado durante as reações exotérmicas. A hipótese de que o calor gerado pela reação química é proporcional à extensão da cura permite obter os parâmetros cinéticos no DSC por meio das análises pelos métodos isotérmicos e não-isotérmicos. Para o método isoconversional isotérmico, a energia de ativação média apresentou valores entre 103 e $72 \mathrm{~kJ} / \mathrm{mol}$, e, para o método isoconversional não-isotérmico um valor médio de $80,5 \mathrm{~kJ} / \mathrm{mol}$. Estes resultados foram comparados

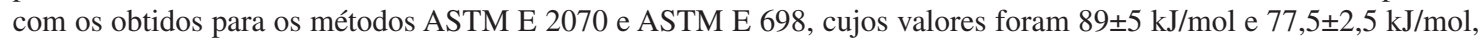
respectivamente, comprovando a eficiência do método isoconversional.
\end{abstract}

Palavras-chave:Cínética de cura, compósito, resina epoxídica, DSC.

\section{Study of Cure Kinetics of a Pre-Impregnated Epoxy Resin/Carbon Fiber Using the Isoconversional Method}

Abstract: This paper aims to determine the kinetics of a pre-impregnated ("prepreg") epoxy resin/carbon fiber using differential scanning calorimetry (DSC) with the isoconversional method. The latter method analyzes the cure kinetics through the dependence of activation energy during conversion (isothermal analysis) or temperature (nonisothermal analysis) and uses this dependence to calculate the kinetic parameters. The evaluation of reaction kinetics by DSC is based on the heat generating rate during an exothermic reaction. The hypothesis that a heat generated by a chemical reaction is proportional to the extension of cure allows one to obtain the kinetic parameters from the DSC curves obtained with the isothermal and nonisothermal modes. For the isoconversional isothermal method, the average activation energy ranged between 72 and $103 \mathrm{~kJ} / \mathrm{mol}$ while in the non-isothermal method an average value of $80.5 \mathrm{~kJ} / \mathrm{mol}$ was obtained. These results were compared with those obtained for ASTM E 2070 and ASTM E 698 methods, whose values were $89 \pm 5 \mathrm{~kJ} / \mathrm{mol}$ and $77.5 \pm 2.5 \mathrm{~kJ} / \mathrm{mol}$, respectively, proving the efficiency of the method.

Keywords: Cure kinetics, composite, epoxy resin, DSC.

\section{Introdução}

A avaliação de parâmetros cinéticos a partir de dados obtidos por meio das técnicas de análises térmica tem sido um dos tópicos bastante explorados nos últimos anos gerando grande número de artigos. Vyazovkin, Rives e Schick (2010) apresentaram uma excelente revisão dos artigos publicados entre 1970 e 2010 na Thermochimica Acta, um dos principais periódicos internacionais sobre análise térmica, sendo que os mais pertinentes são ressaltados a seguir ${ }^{[1]}$.

O maior impacto no campo de análise térmica cinética foi publicado em 1999, no trabalho de Vyazovkin e Wight, que comparou dois modelos computacionais que abordavam avaliações cinéticas: uma aproximação de modelos de reações para dados de taxas de aquecimento simples e uma aproximação isoconversional que elimina o modelo de aproximação em uma única etapa e o substitui usando taxas múltiplas de aquecimento ${ }^{[1,2]}$.

Os resultados obtidos no projeto de cinética promovido pela ICTAC (International Confederation for Thermal Analysis and Calorimetry), no qual vários participantes aplicaram diversas técnicas livres de modelo que empregavam dados de múltiplos estágios isotérmicos e não-isotérmicos obtidos a diferentes temperaturas e/ou várias taxas de aquecimento, foram muito satisfatórios comprovando que o método isoconversional livre de modelo era consistente ${ }^{[3]}$. A mensagem mais importante 
deste projeto é que um método cinético computacional confiável deve usar dados múltiplos obtidos a partir de programas a diferentes temperaturas, enquanto métodos baseados em dados de apenas um aquecimento devem ser evitados $^{[1]}$.

Sbirrazzuoli e Vyazovkin (2002) estudaram a cura de resina epoxídica por meio da análise isoconversional livre de modelo usando dados DSC isotérmicos e nãoisotérmicos e comprovaram a dependência entre a energia de ativação e a extensão da reação de cura ${ }^{[4]}$.

Starink (2003) comparou a precisão de métodos isoconversionais e forneceu um guia prático. O método isoconversional fornece a energia de ativação sem determinar o mecanismo de reação, porém, o mesmo pode ser determinado usando alguns procedimentos computacionais, como o descrito por Malek (1972), ainda bastante utilizado ${ }^{[1]}$.

Sbirrazzuolli et al. (2006) apresentaram uma análise isoconversional da reação estequiométrica e nãoestequiométrica da reação de cura de resina epoxídicaamina, confirmando o grande potencial do método ${ }^{[5]}$.

As mudanças de temperaturas podem estimular uma variedade de processos químicos e físicos em um sistema polimérico. Alguns exemplos importantes desses processos incluem degradação térmica, cura, cristalização, temperatura de transição vítrea, etc. A cinética desses processos pode ser acompanhada por meio dos métodos de análise térmica: análise termogravimétrica (TG), a calorimetria exploratória diferencial (DSC) e outras técnicas ${ }^{[6]}$.

A cinética de cura das resinas epoxídicas pode ser acompanhada pela calorimetria exploratória diferencial (DSC). O fluxo de calor medido no DSC é proporcional ao calor liberado e a taxa de cura pode ser expressa pela Equação 1.

$$
\frac{d Q}{d t}=Q_{r x n} \frac{d \alpha}{d t}=Q_{r x n} k(T) f(\alpha)
$$

onde $\mathrm{dQ} / \mathrm{dt}$ é o fluxo de calor, $\mathrm{Q}_{\mathrm{rxn}}$ é o calor total proveniente da cura do material, d $\alpha / d t$ é a taxa de cura, $\alpha$ é a conversão do material, $\mathrm{k}(\mathrm{T})$ é a constante de proporcionalidade, $\mathrm{T}$ é a temperatura, $\mathrm{f}(\alpha)$ é o modelo da reação. Para resinas epoxídicas, $\mathrm{f}(\alpha)$ é usualmente apresentada na forma de $(1-\alpha)^{\mathrm{n}}$ (reação de ordem $\mathrm{n}$ ) ou $\alpha^{\mathrm{m}}(1-\alpha)^{\mathrm{n}}$ (cura autocatalítica).

A dependência da temperatura com a constante é introduzida substituindo k(T) pela equação de Arrhenius:

$$
\frac{d \alpha}{d t}=A \exp \left(-\frac{E}{R T}\right) f(\alpha)
$$

onde, $\mathrm{R}$ é a constante ideal dos gases, $\mathrm{T}$ é a temperatura, E é a energia de ativação e A é denominado fator de freqüência. $\mathrm{O}$ fator de freqüência relaciona o número de colisões que são necessárias por unidade de tempo para ocorrer a reação, a energia de ativação descreve a quantidade de energia necessária para propagar a reação.

Para processos não-isotérmicos, nos quais uma amostra é aquecida a razão constante, a dependência do tempo presente na Equação 2 é substituída resultando na Equação 3.

$$
\frac{d \alpha}{d T}=\left(\frac{A}{\beta}\right) \exp \left(-\frac{E}{R T}\right) f(\alpha)
$$

onde $\beta=\mathrm{dT} / \mathrm{dt}$ é a taxa de aquecimento. Para as condições isotérmicas e não-isotérmicas, a extensão da conversão, $\alpha$, é calculado pela integração da área sob os picos de DSC.

Existem duas aproximações (hipóteses) na análise cinética. A primeira está relacionada com o mecanismo de reação que se assume. Comumente os dados são ajustados para o modelo de reação de uma única etapa, o que naturalmente produz um único valor de energia de ativação para todo o processo de cura. O resultado não reflete mudanças no mecanismo de reação e nem na cinética com a temperatura e a extensão da conversão. Entretanto, sabe-se que a cura das resinas epoxídicas envolve múltiplas etapas que provavelmente tenham diferentes energias de ativação. A contribuição destas etapas na taxa de cura total geralmente deve variar com a temperatura e a extensão da reação de cura. O que significa que a energia de ativação determinada pela medida da taxa de cura total é função destas duas variáveis. Este tipo de modelo é muito ineficiente na detecção de reações com este grau de complexidade. Além disso, quando aplicadas para dados de taxas de aquecimento não-isotérmicos, esta aproximação tende a produzir valores incertos de energia de ativação, os quais não podem ser comparados com os valores de dados isotérmicos ${ }^{[4]}$.

Uma alternativa é utilizar o método isoconversional livre de modelo, o qual não assume um mecanismo de reação particular, porém, permite avaliar a energia de ativação como função da extensão da cura. O tratamento isoconversional dos dados de análise térmica foi reavivado fortemente por Vyazovkin (1997, 1999), que desenvolveu um avançado método isoconversional não linear que evita imprecisões associadas com aproximações analíticas da integral da temperatura. Este tipo de análise permite não somente obter resultados consistentes pelos métodos isotérmicos e não-isotérmicos, mas também proporciona importantes informações a respeito do mecanismo de $\operatorname{cura}^{[4]}$.

As mudanças no mecanismo de reação estão associadas às mudanças na energia de ativação, que podem ser detectadas usando o método isoconversional livre de modelo. Este método está baseado no princípio isoconversional no qual a taxa de reação em uma extensão constante de conversão é apenas função da temperatura, conforme a Equação 4.

$$
\left[\frac{d \ln (d \alpha / d t)}{d T^{-1}}\right]_{\alpha}=-\frac{E}{R}
$$

$\mathrm{O}$ índice $\alpha$ indica o valor relacionado a uma certa extensão da conversão. Este fato preside apenas uma reação de uma única etapa. Para reações de cinética de múltiplas etapas, o valor total de $\alpha$ não é exclusivamente determinado pela composição da amostra. Neste último caso, $\mathrm{E}$ varia com $\alpha$, o que reflete a variação na contribuição relativa das etapas únicas na taxa de reação total $^{[4]}$.

O método isoconversional emprega um programa de múltiplas temperaturas, ou seja, diferentes taxas de aquecimento e/ou temperaturas, porque esta é 
praticamente a única maneira de obter dados variando as taxas a uma extensão de reação constante de conversão, isto é, o lado esquerdo da Equação 4. Observar que a Equação 4 é derivada de uma equação cinética de uma única etapa (Equação 2). Entretanto, a suposição principal do método isoconversional de uma única etapa (Equação 1), é aplicável apenas a conversões de extensão simples e a regiões de temperatura $(\Delta \mathrm{T})$ relacionadas com a conversão (Figura 1).

Em outras palavras, o método isoconversional descreve a cinética do processo usando múltiplas equações de uma única etapa, cada uma associada a uma certa extensão da conversão no processo de cura (Figura 1).

O rearranjo da Equação 2 leva a seguinte equação:

$$
\ln \left(\frac{d \alpha}{d t}\right)_{\alpha, i}=\ln \left[\mathrm{A}_{\alpha} \mathrm{f}(\mathrm{x})\right]-\frac{E_{\alpha}}{R T_{\alpha, i}}
$$

Onde o $\mathrm{i}$ indica as diferentes taxas de aquecimento.

Para condições isotérmicas, a integração da Equação 2 tem-se:

$$
g(\alpha) \equiv \int_{0}^{\alpha} \frac{d \alpha}{f(\alpha)}=A \exp \left(\frac{-E}{R T}\right) t
$$

onde $\mathrm{g}(\alpha)$ é a forma integral do modelo de reação. Rearranjando a Equação 6 tem-se:

$$
-\ln t_{\alpha, i}=\ln \frac{A_{\alpha}}{g_{(\alpha)}}-\frac{E_{\alpha}}{R T_{i}}
$$

que pode ser usada para determinar a dependência de $\mathrm{E} \alpha$ nas corridas isotérmicas executadas em diferentes temperaturas $\mathrm{T}_{\mathrm{i}}$.

Para experimentos não-isotérmicos, quando a temperatura é atingida a uma razão constante de aquecimento $\beta$, a integração da Equação 2 envolve resolução da integral da temperatura $\mathrm{I}(\mathrm{E}, \mathrm{T})$

$$
g(\alpha)=\frac{A}{\beta} \int_{0}^{T} \frac{-E}{R T} d T=\frac{A}{\beta} I(E, T)
$$

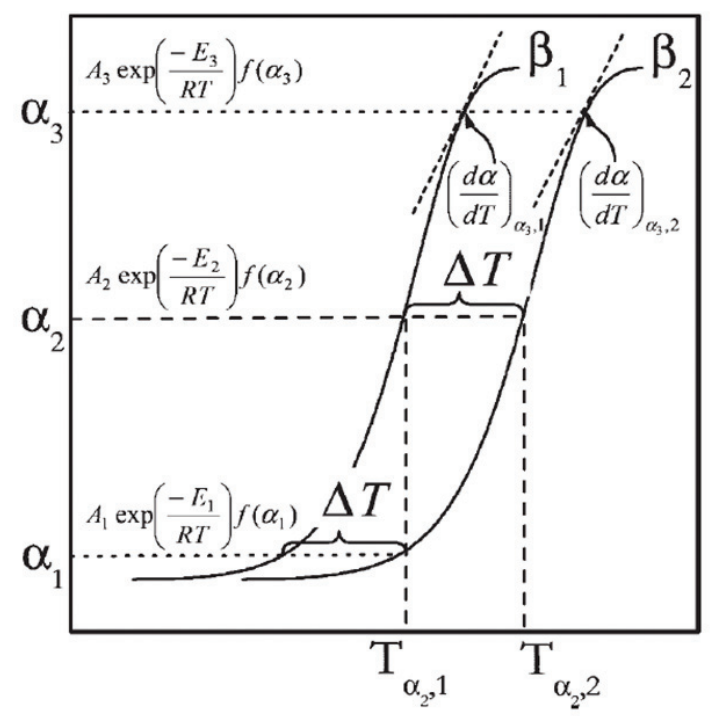

Figura 1. Aplicação da Equação de Arrhenius pelo Método Isoconversional numa faixa estreita de temperatura ${ }^{[4]}$.
Para um conjunto de valores de $\mathrm{n}$ experimentos realizados em diferentes $\beta$, E pode ser determinada para cada $\alpha$, calculando-se o valor de E $\alpha$ para o qual a equação Equação 8 tem um mínimo, resultando:

$$
\begin{aligned}
& \sum_{i=l j \neq i}^{n} \sum_{j \neq i}^{n} \frac{I\left(E_{\alpha}, T_{\alpha, i}\right) \beta_{j}}{I\left(E_{\alpha} T_{\alpha, j}\right) \beta_{i}} \\
& I\left[E_{\alpha}, T_{i}\left(t_{\alpha}\right)\right]=\int_{t_{1}}^{t_{2}} \exp \left[\frac{E_{\alpha}}{R T_{i}(t)}\right] d t
\end{aligned}
$$

O procedimento de minimização é repetido para cada valor de $\alpha$ para encontrar a dependência da energia de ativação com a extensão da conversão.

Rearranjando a Equação 6 tem-se:

$$
t_{\alpha}=\frac{g_{(\alpha)}}{\operatorname{Aexp}\left(-\frac{E}{R T_{0}}\right)}
$$

A avaliação exclusiva da dependência de E$\alpha$ é suficiente para prever a cinética isotérmica a partir de dados não isotérmicos. Isto é formalizado pela Equação 12 que possibilita estimar o tempo a partir do qual uma dada conversão, em uma temperatura arbitrária, $\mathrm{T}_{0}$, será alcançada.

$$
t_{\alpha}=\frac{\int_{0}^{T_{\alpha}} \exp \left(-\frac{E}{R T}\right) d T}{\beta \exp \left(-\frac{E_{\alpha}}{R T_{0}}\right)}
$$

onde $\mathrm{T}_{\alpha}$ é a temperatura na qual uma determinada conversão é atingida numa taxa de aquecimento $\beta$.

Os métodos de análise cinética isoconversionais já são conhecidos de longa data, tais como os propostos por Friedman (1964), Ozawa (1965) ou Flynn e Wall (1966) e possibilitam revelar a dependência da energia de ativação com a extensão da conversão e com a temperatura ${ }^{[2]}$.

Todo esse processo descrito acima (Equação 9 e Equação 12) pode ser simplesmente resumido a aplicar o método proposto por Ozawa, Flynn e Wall, ASTM E $698^{[7]}$, ou o método isotérmico, ASTM E $2070^{[8]}$, em toda a faixa de conversão e não somente de forma pontual, como no máximo de picos DSC ou DTG (termogravimetria derivada) e, em seguida, traçar o gráfico das energias de ativação obtidas em função do grau de conversão. Nos processos regidos por apenas uma energia de ativação, as retas obtidas serão paralelas, caso contrário, várias inclinações poderão ser observadas, o que pode ser muito útil em estabelecer mecanismos de reação.

A análise cinética pode ter dois propósitos diferentes, um teórico e outro prático. O principal propósito prático é a previsão das taxas de cura de processamento ou tempo de vida útil do material. As previsões serão confiáveis dependendo do método cinético aplicado ${ }^{[3]}$.

Com base no que foi exposto, este trabalho tem por objetivo avaliar a cinética de cura de um préimpregnado ("prepreg") de resina epoxídica/fibra de carbono de aplicação aeronáutica, utilizando a técnica de calorimetria diferencial de varredura (DSC) pelo método isoconversional, em condições isotérmicas e nãoisotérmicas. 


\section{Experimental}

\section{Material}

Foram utilizados dois tipos de "prepregs" de uso aeronáutico com a mesma matriz de resina epoxídica, porém, com diferentes tipos de reforços, fibra de carbono (V01X) e fibra de vidro (V02X), este apenas como referência para quantificar o teor de resina no V01X.

\section{Equipamentos utilizados}

Para as análises, foram utilizados os seguintes equipamentos:

- Calorímetro Exploratório Diferencial, DSC-910 e Analisador Termogravimétrico TGA-951, ambos da Du Pont com o Analisador Térmico TA2100 TA Instruments.

\section{Métodos}

As análises DSC pelo método não-isotérmico foram realizadas em diferentes razões de aquecimento $3,5,10$, 15 e $20^{\circ} \mathrm{C} / \mathrm{min}$. Para o método isotérmico as temperaturas de cura (Tc) foram de 100,110 e $120^{\circ} \mathrm{C}$, abaixo da temperatura de transição vítrea da resina curada $(\mathrm{Tg} \cong$ $126^{\circ} \mathrm{C}$ ) do material ${ }^{[9]}$.

Em ambos os métodos, as análises foram realizadas em porta amostra de alumínio hermético $( \pm 20 \mathrm{mg}) \mathrm{sob}$ fluxo de $50 \mathrm{ml} / \mathrm{min}$ de nitrogênio que proporcionou uma atmosfera inerte.

As análises de TG consistiram no aquecimento da amostra a partir da temperatura ambiente numa razão de aquecimento a $20^{\circ} \mathrm{C} / \mathrm{min}$ até atingir $620^{\circ} \mathrm{C}$, mantendo em isoterma por um período de $40 \mathrm{~min}$, sob atmosfera de nitrogênio ${ }^{[9,10]}$.

\section{Resultados e Discussão}

A análise termogravimétrica (TG) teve como objetivo definir a estabilidade térmica e a obtenção do teor de resina do "prepreg".

O teor obtido para o "prepreg" V01X por TG foi de $37 \pm 1 \%$ e os valores de entalpias de cura obtidos na análise DSC foram corrigidos por este valor ${ }^{[9]}$.

As curvas DSC obtidas para o "prepreg" V01X pelo método não-isotérmico são mostradas na Figura 2. A partir da integração da área sob o pico da curva DSC obteve-se a entalpia total envolvida no processo de cura da resina, que foi corrigido pelo teor de resina obtido na análise $\mathrm{TG}^{[9]}$.

A extensão da conversão, no caso de acompanhamento por DSC, é calculada por $\alpha=\Delta \mathrm{Hc} / \Delta \mathrm{Ht}$, onde $\Delta \mathrm{Hc}$ é a entalpia parcial na temperatura $\mathrm{T}$, obtida por meio da integração da área abaixo da curva até temperatura T. A entalpia total da reação, $\Delta \mathrm{Ht}$, foi calculada por meio da integração da área do pico em cada razão de aquecimento.

Para a aplicação do método não-isotérmico foi gerado o gráfico da extensão da conversão $(\alpha)$ x temperatura para as diferentes razões de aquecimento (Figura 3). A partir destas curvas foi possível extrair os dados que relacionam as temperaturas necessárias em diferentes razões de aquecimento $(\beta)$ para se atingir o grau de conversão específico. Com estes dados foi gerado o gráfico $\ln \beta \times$

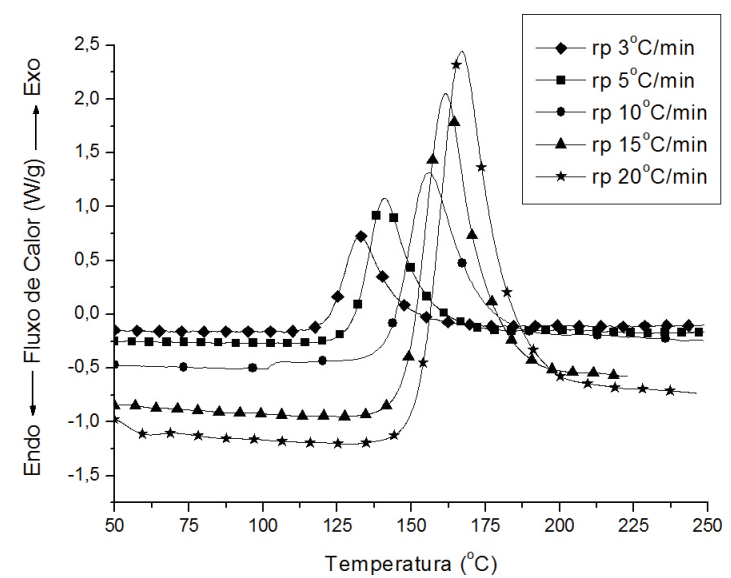

Figura 2. Análise calorimétrica diferencial (DSC): Curvas da cura não-isotérmica.

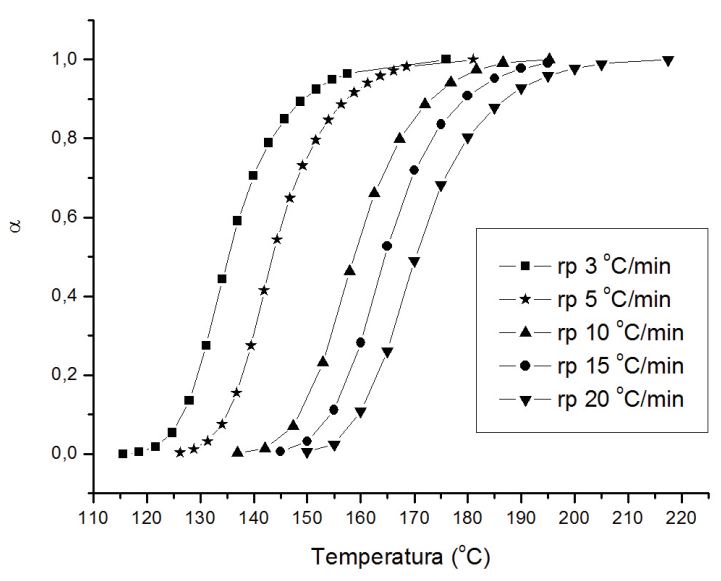

Figura 3. Método isoconversional não-isotérmica: $\alpha \quad x$ Temperatura $\left({ }^{\circ} \mathrm{C}\right)$.

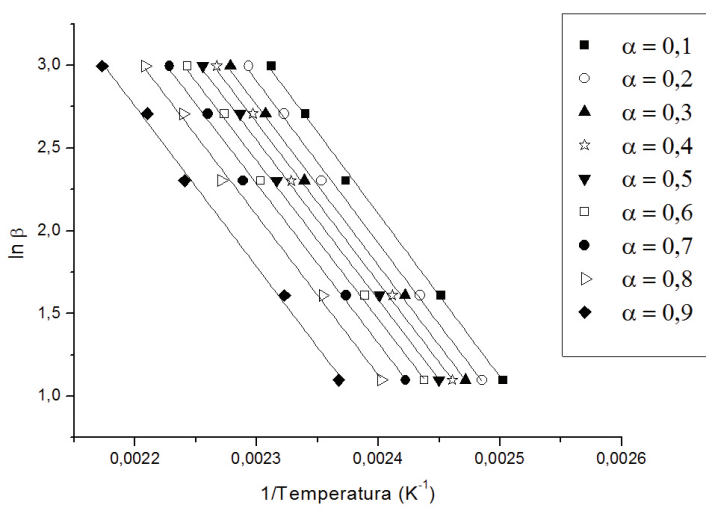

Figura 4. Método isoconversional não-isotérmico: $\ln \beta \times 1 /$ Temperatura $\left(\mathrm{K}^{-1}\right)$.

1/temperatura (Figura 4), e, aplicando-se a regressão linear, calculou-se a energia de ativação para cada grau de conversão (Figura 5).

As análises de cura no DSC (Figura 2) também foram utilizadas para calcular os parâmetros cinéticos pelo método ASTM E 698, e assim gerar dados para comparar com os resultados obtidos pelo método isoconversional 
não-isotérmico. O parâmetro escolhido para se determinar a energia de ativação foi a temperatura no máximo do pico em cada razão de aquecimento (Tabela 1). A partir do gráfico construído de $\ln \beta \times 1 /$ Temperatura obtém-se os valores dos parâmetros cinéticos.

Para o método isoconversional não-isotérmico observa-se uma energia de ativação que se apresenta quase constante a $80,5 \mathrm{~kJ} / \mathrm{mol}$ (Figura 5), valor próximo ao encontrado por meio da aplicação do método ASTM E 698 que é de $77,5 \pm 2,5 \mathrm{~kJ} / \mathrm{mol}$.

As análises de cura no DSC isotérmicas nas temperaturas de 100,110 e $120^{\circ} \mathrm{C}$ foram realizadas colocando-se as amostras dentro do forno do equipamento após ser atingida a temperatura de análise requerida (Figura 6). Da integração da área sob o pico da curva

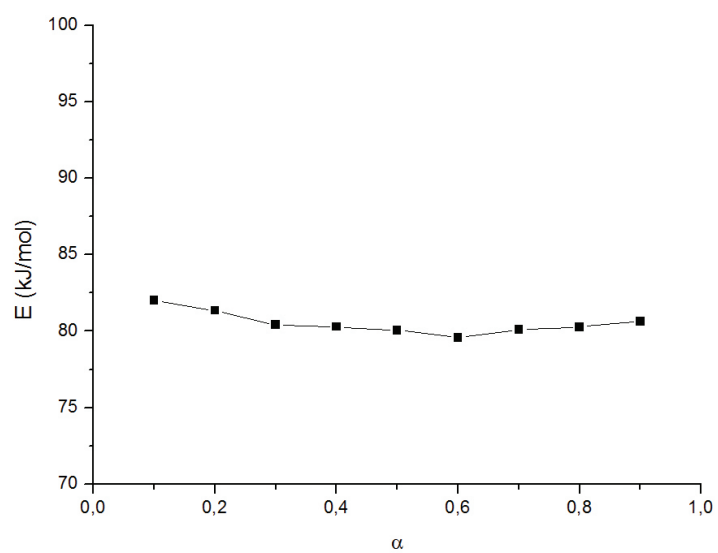

Figura 5. Método isoconversional não-isotérmico: $\mathrm{E}(\mathrm{kJ} / \mathrm{mol}) \mathrm{x} \alpha$.

Tabela 1. Valores das razões de aquecimento e a temperatura no máximo do pico DSC

\begin{tabular}{ccc}
\hline$\beta$ & $\begin{array}{c}\mathbf{T}_{\mathbf{1}} \\
(\mathbf{K})\end{array}$ & $\begin{array}{c}\mathbf{T}_{2} \\
(\mathbf{K})\end{array}$ \\
\hline 3 & 405,4 & - \\
5 & 414,1 & 413,7 \\
10 & 429,1 & 429,0 \\
15 & 434,5 & 434,9 \\
20 & 440,0 & - \\
\hline
\end{tabular}

1 referente à $1^{\circ}$ corrida; 2 referente à duplicata.

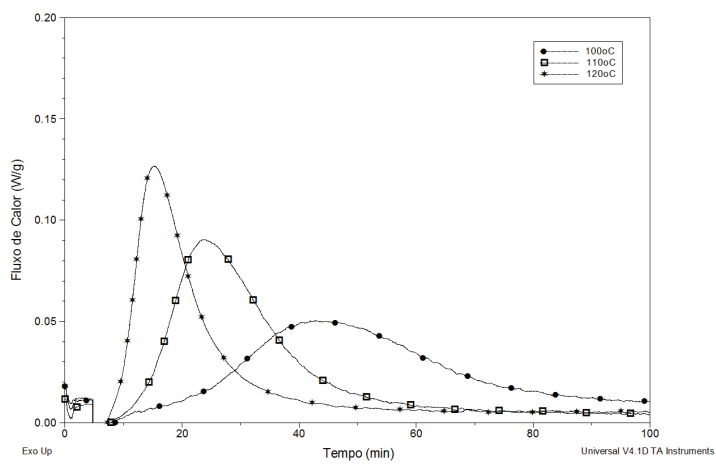

Figura 6. Análise calorimétrica diferencial (DSC): Curvas da cura isotérmica.
DSC obteve-se a entalpia total envolvida no processo de cura da resina.

A extensão da conversão foi calculada por $\alpha=\Delta \mathrm{Hc} / \Delta \mathrm{Ht}$, onde $\Delta \mathrm{Hc}$ é a entalpia parcial no tempo t, obtida por meio da integração da área abaixo da curva até o tempo t. A entalpia total da reação, $\Delta \mathrm{Ht}$, é calculada por meio da integração da área sob os picos.

Construiu-se o gráfico de extensão da conversão $(\alpha) \mathrm{x}$ tempo (min) para as diferentes temperaturas de isotermas (Figura 7). A partir destas curvas extraíram-se os dados que relacionam os diferentes tempos necessários em cada isoterma para se atingir o grau de conversão específico. Com estes dados traça-se o gráfico ln (tempo) $\mathrm{x}$ 1/Temperatura da isoterma para cada grau de conversão (Figura 8) e calcula-se a energia de ativação para cada grau de conversão (Figura 9).

No método isoconversional isotérmico (Figura 9) observa-se uma queda de energia de ativação ao longo do processo de cura que varia de 103 a $72 \mathrm{~kJ} / \mathrm{mol}$ e ainda é possível visualizar o processo de desaceleração da reação de cura que apresenta seu máximo de taxa de conversão no início do processo e diminui constantemente conforme maiores valores de conversão são alcançados ${ }^{[3]}$.

As análises de cura isotérmicas no DSC também foram utilizadas para calcular os parâmetros cinéticos pelo método ASTM E 2070, e assim gerar dados para comparar

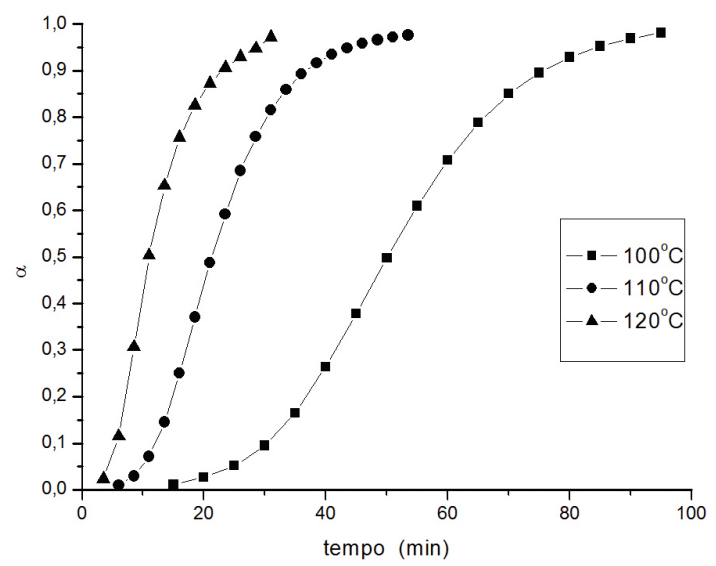

Figura 7. Método isoconversional isotérmico: $\alpha \times$ tempo (min).

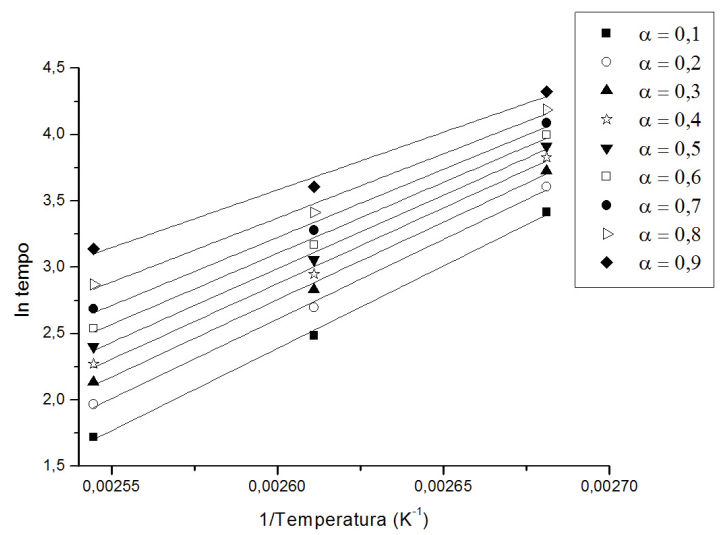

Figura 8. Método isoconversional isotérmico: ln tempo $\mathrm{x} \times 1$ / Temperatura $\left(\mathrm{K}^{-1}\right)$. 


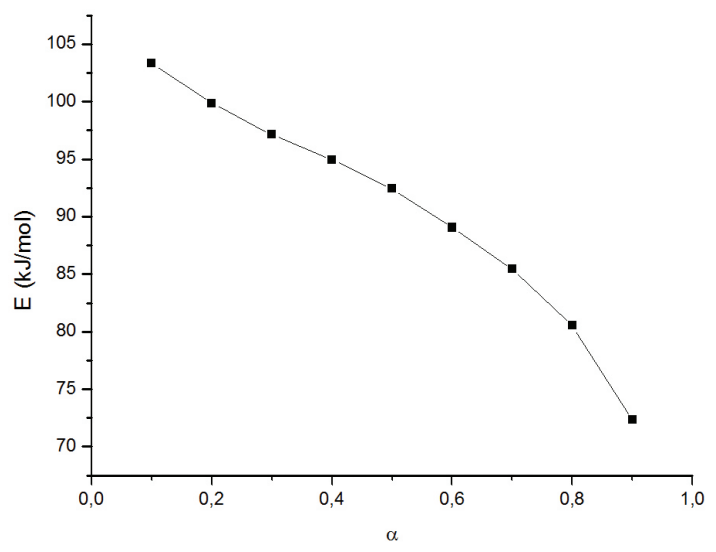

Figura 9. Método isoconversional isotérmico: $\mathrm{E}(\mathrm{kJ} / \mathrm{mol})$ x $\alpha$.

Tabela 2. Tempo para se atingir o máximo do pico DSC.

\begin{tabular}{cccc}
\hline $\mathbf{T}$ & $\mathbf{T}$ & $\begin{array}{c}\text { Tempo 1 } \\
(\mathbf{m i n})\end{array}$ & $\begin{array}{c}\text { Tempo 2 } \\
(\mathbf{m i n})\end{array}$ \\
\hline 100 & $(\mathbf{K})$ & 27,4 & 38,4 \\
110 & 373 & 12,4 & 16,4 \\
120 & 383 & 6,9 & 8,2 \\
\hline
\end{tabular}

1 referente à $1^{\circ}$ corrida; 2 referente à duplicata.

com os resultados obtidos pelo método isoconversional isotérmico. O parâmetro escolhido para se determinar a energia de ativação, foi o tempo necessário para cada isoterma atingir o ponto máximo do pico (Tabela 2). Os tempos utilizados nos cálculos foram extraídos das curvas isotérmicas a 100,110 e $120^{\circ} \mathrm{C}$ (Figura 6). Neste caso foi descontado o tempo até a abertura do forno para a colocação da amostra. A partir destes dados traçou-se o gráfico de ln (tempo) e 1/Temperatura de cura e obteve-se o valor de energia de ativação médio de $89 \pm 5 \mathrm{~kJ} / \mathrm{mol}$.

\section{Conclusão}

O método isoconversional foi aplicado nas condições isotérmica e não-isotérmica para o acompanhamento do processo de cura do "prepreg" de resina epoxídica e fibra de carbono. No método isoconversional isotérmico foi possível observar a diminuição da energia de ativação de 103 a $72 \mathrm{~kJ} / \mathrm{mol}$ com o avanço do processo de cura, este fato está relacionado com o processo de desaceleração da reação química. Esta valor de energia de ativação pode ser comparado ao valor obtido mediante o método ASTM E 2070 que é de $89 \pm 5 \mathrm{~kJ} / \mathrm{mol}$, valor próximo ao encontrado de energia de ativação no método isoconversional isotérmico com razão de conversão $\alpha=0,60$.

No método isoconversional não-isotérmico não foi possível observar uma nítida alteração da energia de ativação durante o processo de cura, logo adotou-se um valor médio de $80,5 \mathrm{~kJ} / \mathrm{mol}$, valor próximo ao encontrado por meio da aplicação do método ASTM E 698 que é de $77,5 \pm 2,5 \mathrm{~kJ} / \mathrm{mol}$.

Foi possível comprovar o motivo pelo qual métodos que usam múltiplas taxas de aquecimento ou várias temperaturas de cura são recomendados para a avaliação dos parâmetros cinéticos. No método isoconversional isotérmico foi possível detectar a diminuição da energia de ativação no decorrer do processo de cura, o que também confirma que o mecanismo de reação da resina do compósito não ocorre em uma única etapa, mas em múltiplas etapas, como é característico de resina epoxídica. No entanto, o método isoconversional nãoisotérmico não apresentou tal sensibilidade à mudança de energia de ativação no decorrer do processo de cura, apesar do valor encontrado estar dentro da faixa de energia ativação dos demais métodos como o ASTM E 698 ou até mesmo o isoconversional isotérmico.

\section{Referências Bibliográficas}

1. Vyazovkin, S.; Rives, V. \& Schick, C. - Thermochim. Acta, 500, p.1-5 (2010). http://dx.doi.org/10.1016/j. tca.2010.02.005

2. Vyazovkin, S. \& Wight, C. A. - Chem. Mater., 2, p.3386 (1999). http://dx.doi.org/10.1021/cm9904382

3. Vyazovkin, S. - Thermochim. Acta, 355, p.155 (2000). http://dx.doi.org/10.1016/S0040-6031(00)00445-7

4. Sbirrazzuoli, N. \& Vyazovkin, S. - Thermochim. Acta, 388, p.289 (2002). http://dx.doi.org/10.1016/S00406031(02)00053-9

5. Sbirrazzuoli, N.; Mija, A. M.; Vincent, L. \& Alzina, C. - Thermochim. Acta, 447, p.167 (2006). http://dx.doi. org/10.1016/j.tca.2006.06.005

6. Sbirrazzuoli, N. \& Vyazovkin, S. - Macromol. Radid Comun., 27, p.1515 (2006). http://dx.doi.org/10.1002/ marc. 200600404

7. Association Standards Testing Materials - "ASTM E698: Standard Test Method for Arrhenius Kinetic Constants for Thermally Unstable Materials Using Differential Scanning Calorimetry and the Flynn/Wall/Ozawa Method", Philadelphia (1999).

8. Association Standards Testing Materials - "ASTM E 2070: Standard test method for Kinetic Parameters by Differential Scanning Calorimetry Using Isotherma Methods", Philadelphia (2000).

9. Ferrari, V. C. G. M.; Lourenço, V. L.; Dutra, R. C. L.; Diniz, M. F.; Azevedo, M. F. P. \& David, L. H. - Polímeros, 22, p.369 (2012). http://dx.doi.org/10.1590/S010414282012005000048

10. Yee, R. Y. \& Stephens, T. S. - Thermochim. Acta, 520, p.1 (1996).

Enviado: 03/11/12 Reenviado: 27/05/13 Aceito: 04/06/13 